\title{
Aspectos vegeto-produtivos de mirtileiros 'Briteblue' em diferentes formas de propagação e intensidades de poda
}

\author{
Vegetative and productive aspects of 'Briteblue' blueberries in different forms of propagation and pruning \\ intensities
}

\section{Samila Silva Camargo ${ }^{1 *}$, André Luiz Kulkamp de Souza ${ }^{2}$, Laura Reisdörfer Sommer ${ }^{3}$, Márcia Wulff Schuch $^{3}$ e Márcia Vizzotto ${ }^{4}$}

\section{RESUMO}

Ademanda por mirtilos tem aumentado principalmente devido ao seu valor agregado e possibilidade de industrialização. Apesar da grande importância, são necessários estudos que priorizem maior qualidade de mudas e frutos, além de técnicas adequadas de manejo. Estudou-se formas de propagação (micropropagação e estaquia) e intensidades de poda (testemunha, leve e drástica) de mirtileiros 'Briteblue'. Concluímos que plantas micropropagadas, apresentam maior crescimento vegetativo, assim como a poda drástica proporciona o desenvolvimento de ramos vegetativos de maior comprimento. Maiores níveis de antocianinas foram encontrados em frutos oriundos da estaquia e as plantas sem poda ou com poda leve apresentam maior atividade antioxidante.

PALAVRAS-CHAVE: Vaccinium sp., mudas, manejo, pós-colheita.

\footnotetext{
ABSTRACT

The demand for blueberries has increased mainly due to the added value and the possibility of industrialization. Despite the great importance, studies are needed that focus on higher quality of seedlings and fruits, as well as appropriate plant management techniques. We studied propagation forms (micropropagation and cuttings) and pruning intensities (control, light and drastically) of blueberries 'Briteblue'. We concluded that micropropagated plants have an increased vegetative growth, as well as severe pruning provides the development of vegetative branches of greater nutracêuticas.

${ }^{1}$ Universidade do Estado de Santa Catarina, Lages, SC, Brasil.

${ }^{2}$ Empresa de Pesquisa Agropecuária e Extensão Rural de Santa Catarina, Videira, SC, Brasil.

${ }^{3}$ Universidade Federal de Pelotas, Pelotas, RS, Brasil.

${ }^{4}$ Empresa Brasileira de Pesquisa Agropecuária, Pelotas, RS, Brasil.

*Autor para correspondência<samilasc@yahoo.com.br>
}

length. Higher levels of anthocyanins were found in fruits propagated by cuttings and plants without pruning or light pruning had higher antioxidant activity.

KEYWORDS: Vaccinium sp., seedlings, management, post-harvest.

No Brasil, a área plantada de mirtileiro ainda é pequena, principalmente devido à qualidade e preço das mudas, situação decorrente da dificuldade de propagação da maioria das cultivares (TREVISAN et al. 2008). Sendo assim, os estudos realizados com a cultura estão relacionados principalmente com métodos de propagação, a fim de estabelecer a técnica mais adequada para cada grupo ou cultivar (SCHUCH et al. 2007, TREVISAN et al. 2008, DAMIANI \& SCHUCH et al. 2009), e de póscolheita e análise fitoquímica dos frutos (MORAES et al. 2007, BRACKMANN et al. 2010), já que o mirtilo é conhecido mundialmente por suas propriedades

De acordo com VIZZOTTO (2012), estudos que correlacionam o consumo das pequenas frutas e seus benefícios para a saúde estão se intensificando consideravelmente nos últimos anos. Nesse sentido, o mirtilo é uma dessas frutas de maior destaque, devido a sua capacidade antioxidante e presença de compostos fenólicos e antociânicos e por isso, desperta a atenção tanto dos consumidores, quanto dos próprios produtores, pois a cultura pode ser uma nova alternativa para geração de renda nas propriedades.

Trabalhos a campo e de manejo de pomar são ainda escassos, deixando aberta uma lacuna para 
o sucesso da cultura no país (SOUZA et al. 2011). Logo, torna-se necessário o estudo do manejo do pomar e principalmente, da poda de mirtileiros nas condições da região Sul do Brasil, haja vista que os produtores utilizam critérios validados em outros países, o que não é adequado, já que nossas condições edafoclimáticas são bastante distintas.

Objetivou-se a obtenção de dados vegetativos e produtivos, em função de diferentes métodos de propagação e intensidades de poda na região de Pelotas, RS, para a cultivar de mirtileiro 'Briteblue'.

$\mathrm{O}$ experimento foi realizado no município de

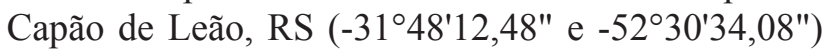
em um pomar de mirtileiros cv. 'Briteblue' instalado em agosto de 2009, com um espaçamento de 1,3 x 4,0 m entre plantas e entre linhas, respectivamente. Foi instalado seguindo um delineamento inteiramente casualizado, já que as plantas foram escolhidas ao acaso em um pomar já estabelecido, em um local com condições uniformes para o desenvolvimento dos mirtileiros. O mesmo foi planejado com um arranjo fatorial 2 × 3, sendo: dois métodos de propagação (micropropagação e estaquia) e três intensidades de poda (testemunha, leve e drástica). Os dados foram submetidos à análise de variância e as médias dos tratamentos comparadas estatisticamente pelo teste de Tukey em nível de 5\% de probabilidade de erro.

No início de agosto dos anos 2013 e 2014, foram realizadas diferentes intensidades de poda nas plantas: sem poda (testemunha), poda leve e poda drástica. A testemunha foi o tratamento no qual não foi realizada poda; a poda leve tratou-se de uma limpeza, onde foram retirados aproximadamente $25 \%$ dos ramos da planta, e a poda drástica caracterizou-se por ser uma poda de forma bastante rigorosa, onde foram retirados cerca de $75 \%$ dos ramos.

Foram realizadas avaliações vegetativas e produtivas, em duas e uma safras, respectivamente, sendo as variáveis analisadas: peso dos ramos podados $(\mathrm{g})$, crescimento de brotações $(\mathrm{cm})$, área da folha $\left(\mathrm{cm}^{2}\right)$, índice de clorofila (unidades SPAD), produção $\left(\mathrm{Kg}_{\text {planta }}{ }^{-1}\right)$, número $(\mathrm{g})$, diâmetro $(\mathrm{mm})$, cor (Comissão Internacional de Iluminação CIE - L* $\left.\mathrm{a}^{*} \mathrm{~b}^{*}\right), \mathrm{pH}$, sólidos solúveis ( ${ }^{\circ}$ Brix), acidez titulável (meq $100 \mathrm{~mL}^{-1}$ ), fenólicos totais ( $\mathrm{mg}$ ácido clorogênico equivalente $/ 100 \mathrm{~g}$ tecido), antocianinas totais (mg cianidina 3-glicosídeo/100 g amostra) e atividade antioxidante dos frutos ( $\mu \mathrm{g}$ trolox equivalente/g tecido).

De acordo com a Tabela 1, verifica-se que nas duas safras de cultivo (2013/14 e 2014/15), as plantas 'Briteblue' micropropagadas podadas drasticamente se diferenciaram dos outros tratamentos, ou seja, as oriundas da técnica de micropropagação exigiram uma maior intensidade de poda do que as originadas por estaquia, devido ao maior crescimento vegetativo destas plantas estimulado pelo rejuvenescimento ocorrido in vitro. Além disso, verificou-se que na segunda safra de avaliação, o peso de ramos foi superior, quando comparado ao primeiro ano de estudo. Esse fato também foi verificado por outros autores, onde à campo, mirtileiros propagados in vitro apresentam maior número de brotações do que as propagadas por estaquia (SOUZA et al. 2011). Em estudos com mirtileiros 'Herbert', as plantas derivadas da cultura de tecidos também cresceram com maior vigor e uniformidade do que plantas oriundas da estaquia durante os primeiros três anos no campo (LITWIÑCZUK et al. 2005).

Para as variáveis área da folha, altura de planta e índice de clorofila não ocorreram interações entre os dois fatores nos dois anos de cultivo (Tabela 2). $\mathrm{O}$ método de propagação, assim como a intensidade de poda não influenciaram a área da folha das plantas. As plantas micropropagadas se desenvolveram mais em altura, nas duas safras, comparada com as propagadas por estaquia e resultados semelhantes foram encontrados por SOUZA et al. (2011), onde verificaram que a propagação in vitro foi a que induziu maior crescimento vegetativo inicial, representada pela maior altura de planta.

No primeiro e segundo anos de avaliações, o método de propagação não alterou o índice de clorofila das folhas, apenas na segunda safra, a intensidade de poda. KADIR (2003) relata que a poda alterou essa característica e as folhas apresentaram maiores taxas de clorofila nas plantas com poda drástica na safra 2013/14, já que a retirada das folhas e ramos dentro da copa aumenta a penetração da luz solar. Além disso, no primeiro ano de estudo, a taxa fotossintética e absorção de clorofila, também foi afetada pelos tratamentos de poda, onde as plantas podadas drasticamente apresentaram maior índice de clorofila nas folhas. JORQUERA-FONTENA et al. (2014) também constataram a influência da poda nesta variável, porém, as plantas podadas levemente tiveram índices mais elevados do que as convencionalmente e drasticamente podadas, indicando que o potencial fotossintético do sol exposto folhas de plantas convencionais e severamente podadas estava 
Tabela 1 - Peso de ramos (g) em diferentes intensidades de poda (testemunha, leve e drástica), em mirtileiros cv. 'Briteblue', propagados de diferentes formas (micropropagação e estaquia). Pelotas, RS, 2016.

Table 1 - Weight of branches ( $g$ ) in different pruning intensities (control, light and drastically) in mirtileiros $\mathrm{cv}$. 'Briteblue', propagated in different ways (micropropagation and cuttings). Pelotas, RS, 2016.

\begin{tabular}{|c|c|c|}
\hline \multirow{2}{*}{ Intensidades de poda } & Micropropagação & Estaquia \\
\hline & \multicolumn{2}{|c|}{$2013 / 14$} \\
\hline Testemunha & $0,00 \mathrm{Ac}$ & $0,00 \mathrm{Ab}$ \\
\hline Leve & $608,67 \mathrm{Ab}$ & $586,00 \mathrm{Aa}$ \\
\hline Drástica & $1494,00 \mathrm{Aa}$ & $813,33 \mathrm{Ba}$ \\
\hline \multirow[t]{2}{*}{ CV (\%) } & \multicolumn{2}{|c|}{23,28} \\
\hline & \multicolumn{2}{|c|}{$2014 / 15$} \\
\hline Testemunha & $0,00 \mathrm{Ab}$ & $0,00 \mathrm{Ab}$ \\
\hline Leve & $478,67 \mathrm{Ab}$ & $445,33 \mathrm{Ab}$ \\
\hline Drástica & $2401,33 \mathrm{Aa}$ & $1492,67 \mathrm{Ba}$ \\
\hline
\end{tabular}

Médias seguidas pela mesma letra minúscula na coluna ou maiúscula na linha não diferem pelo teste de Tukey com nível de significância de 5\%.

Tabela 2 - Área da folha $\left(\mathrm{cm}^{2}\right)$, altura de planta (m) e índice de clorofila (unidades SPAD) de plantas de mirtileiro 'Briteblue' em diferentes formas de propagação, micropropagadas e por estaquia e diferentes intensidades de poda (testemunha, leve e drástica). Pelotas, RS, 2016.

Table 2 - Leaf area $\left(\mathrm{cm}^{2}\right)$, plant height $(\mathrm{m})$ and chlorophyll index (SPAD units) of 'Briteblue' blueberry plants in different forms of propagation and micropropagation for cutting and pruning different intensities (control, light and drastically). Pelotas, RS, 2016.

\begin{tabular}{|c|c|c|c|}
\hline & Área da folha & $\begin{array}{c}\text { Altura de } \\
\text { planta }\end{array}$ & $\begin{array}{l}\text { Índice de } \\
\text { clorofila }\end{array}$ \\
\hline & \multicolumn{3}{|c|}{$2013 / 14$} \\
\hline Micropropagação & $6,70 \mathrm{~ns}$ & $1,44 a^{*}$ & $35,60 \mathrm{~ns}$ \\
\hline Estaquia & $7,00 \mathrm{~ns}$ & $1,22 \mathrm{~b}$ & $38,90 \mathrm{~ns}$ \\
\hline Testemunha & $6,85 \mathrm{~ns}$ & $1,23 \mathrm{~ns}$ & $37,25 \mathrm{~b}$ \\
\hline Leve & $5,98 \mathrm{~ns}$ & 1,23 ns & $37,20 \mathrm{~b}$ \\
\hline Drástica & 6,87 ns & $1,54 \mathrm{~ns}$ & $42,45 \mathrm{a}$ \\
\hline \multirow[t]{2}{*}{$\mathrm{CV}(\%)$} & 20,17 & 16,28 & 5,00 \\
\hline & \multicolumn{3}{|c|}{$2014 / 15$} \\
\hline Micropropagação & 8,06 ns & $1,44 \mathrm{a}$ & $46,28 \mathrm{~ns}$ \\
\hline Estaquia & 7,52 ns & $1,2 \mathrm{~b}$ & $44,52 \mathrm{~ns}$ \\
\hline Testemunha & 7,6 ns & 1,3 ns & $44,98^{\mathrm{ns}}$ \\
\hline Leve & $7,7 \mathrm{~ns}$ & 1,27 ns & $45,48^{\mathrm{ns}}$ \\
\hline Drástica & $7,6^{\mathrm{ns}}$ & $1,39^{\mathrm{ns}}$ & $45,73^{\mathrm{ns}}$ \\
\hline CV (\%) & 22,53 & 14,69 & 9,46 \\
\hline
\end{tabular}

*Significativo pelo teste de Tukey ao nível de 5\% de probabilidade de erro.

${ }^{\text {ns }}$ não significativo. 
operando abaixo do seu potencial máximo.

O desenvolvimento dos ramos, nos dois anos (Tabela 3), tiveram comportamento diferenciado, já que na safra 2013/14 houve interação entre os fatores e as plantas micropropagadas com poda leve, assim como, as obtidas por estaquia e podadas drasticamente, apresentaram ramos vegetativos de maior comprimento. Constata-se com esses resultados, que prática de poda, é muito importante, pois além de manter os ramos mais vigorosos, tende a torná-los mais produtivos posteriormente (PESCIE et al. 2011). E ainda, de acordo com SPIERS et al. (2002), a ausência de poda resulta em frutos pequenos e alta produção, além de baixo crescimento dos novos ramos, que serão responsáveis pela produção no ano seguinte.

No segundo ano, as técnicas de propagação não diferiram estatisticamente entre si, porém, mais uma vez, as plantas podadas drasticamente favoreceram o desenvolvimento dos ramos novos. Esse resultado difere dos estudados por MARINO et al. (2014), onde desde o plantio e após o primeiro e segundo anos de crescimento, as plantas oriundas da cultura de tecidos tiveram ramos de maior comprimento, comparadas com as plantas por estaquia.

Ambos, métodos de propagação e intensidades de poda, em plantas de mirtileiro cv. 'Briteblue', tiveram diferenças para as variáveis diâmetro médio e número de frutos, assim como para a produção de mirtilos (Tabela 4), diferentemente dos resultados encontrados por SOUZA et al. (2011), onde não foi constatada diferença significativa entre os métodos de propagação para as variáveis produção por planta e por hectare, diâmetro dos frutos e número de frutos colhidos por planta. Verifica-se que o diâmetro dos frutos está diretamente relacionado com a intensidade de poda e que as plantas testemunhas (sem poda) nos dois métodos de propagação, produziram frutos de menor calibre, quando comparadas com as podadas.

Entretanto, na mesma Tabela, o número de frutos e a produção por planta foram maiores em plantas micropropagadas sem poda, porém, não diferindo estatisticamente das obtidas por estaquia (podas leve e drástica) e poda leve, respectivamente. Estudos semelhantes com mirtileiros mostraram que as plantas submetidas à poda leve foram mais produtivas, fato que provavelmente esteja associado à permanência de maior número de ramos na planta, convergindo para o maior número de gemas floríferas, consequentemente apresentando o maior número de frutos (RADÜNZ et al. 2014). JORQUERA-FONTENA et al. (2014) constataram que a poda em plantas de mirtilo 'Brigitta' estimulou uma maior produtividade e também, um maior número de frutos por planta.

Tabela 3 - Comprimento dos ramos $(\mathrm{cm})$ de plantas 'Briteblue' em diferentes formas de propagação, micropropagadas e por estaquia e diferentes intensidades de poda (testemunha, leve e drástica), nas safras 2013/14 e 2014/15. Pelotas, RS, 2016.

Table 3 - Length of branches $(\mathrm{cm})$ 'Briteblue' plants in different forms of propagation, micropropagation and cuttings and different pruning intensities (control, light and drastically) in 2013/14 and 2014/15 seasons. Pelotas, RS, 2016.

\begin{tabular}{|c|c|c|c|c|c|}
\hline \multicolumn{6}{|c|}{ Comprimento dos ramos } \\
\hline \multicolumn{3}{|c|}{$2013 / 14$} & \multicolumn{3}{|c|}{$2014 / 15$} \\
\hline \multirow{2}{*}{$\begin{array}{c}\text { Intensidade } \\
\text { de poda }\end{array}$} & \multicolumn{2}{|c|}{ Método de propagação } & \multirow{2}{*}{$\begin{array}{l}\text { Método de } \\
\text { propagação }\end{array}$} & \multirow{2}{*}{$\begin{array}{c}\text { Micropropagação } \\
\text { Estaquia }\end{array}$} & \multirow{2}{*}{$\begin{array}{l}26,44^{\text {ns }} \\
29,74 \text { ns }\end{array}$} \\
\hline & Micropropagação & Estaquia & & & \\
\hline Testemunha & $16,67 \mathrm{Ab}$ & $11,62 \mathrm{Ab}$ & \multirow{3}{*}{$\begin{array}{c}\text { Intensidade } \\
\text { de poda }\end{array}$} & Testemunha & $23,00 b^{*}$ \\
\hline Leve & $24,75 \mathrm{Aa}$ & $17,08 \mathrm{Bab}$ & & Leve & $23,22 \mathrm{~b}$ \\
\hline Drástica & $17,25 \mathrm{Aab}$ & $23,33 \mathrm{Aa}$ & & Drástica & $38,05 \mathrm{a}$ \\
\hline CV (\%) & 19,14 & & & CV (\%) & 16,05 \\
\hline
\end{tabular}

*Médias seguidas pela mesma letra minúscula na coluna ou maiúscula na linha não diferem pelo teste de Tukey com nível de significância de 5\%.

*Significativo pelo teste de Tukey ao nível de $5 \%$ de probabilidade de erro.

${ }^{\text {ns }}$ não significativo. 
Em relação às variáveis apresentadas naTabela 5, a intensidade de poda influenciou apenas a acidez titulável de mirtileiros 'Briteblue', onde as plantas sem poda produziram frutos de maior acidez, comparadas às podadas. SOUZA et al. (2014) verificaram que a acidez titulável foi influenciada pela prática da poda no ciclo 2011/2012.

Nesse estudo, não houve diferença significativa entre os tratamentos quanto ao $\mathrm{pH}$ dos frutos, em contrapartida, SOUZA et al. (2011) notaram diferença entre os métodos de propagação para a mesma cultivar, em que foi possível constatar menor valor nos frutos de plantas micropropagadas. Essa resposta pode ser explicada pela existência de maior proporção de casca em relação à polpa, como característica da variedade (GIOVANELLI \& BURATTI 2009). A diferença estatística também não ocorreu no peso médio de frutos, tanto para o método de propagação, quanto para a intensidade de poda, contrariando resultados obtidos por JORQUERA-FONTENA et al. (2014),

Tabela 4 - Diâmetro médio dos frutos $(\mathrm{mm})$, número de frutos e produção (Kg planta-1 $\left.{ }^{-1}\right)$ de plantas de mirtileiro 'Briteblue' em diferentes formas de propagação, micropropagadas (MP) e por estaquia (EST) e diferentes intensidades de poda (testemunha, leve e drástica). Pelotas, RS, 2016.

Table 4 - Average diameter of fruit ( $\mathrm{mm}$ ), number of fruits and production ( $\mathrm{kg} \mathrm{plant}^{-1}$ ) plant blueberry 'Briteblue' in different forms of propagation, micropropagation (MP) and cutting (EST) and different pruning intensities (control, light and drastically). Pelotas, RS, 2016.

\begin{tabular}{|c|c|c|c|c|c|c|}
\hline \multirow{3}{*}{$\begin{array}{c}\text { Intensidade } \\
\text { de poda }\end{array}$} & \multicolumn{2}{|c|}{ Diâmetro médio dos frutos } & \multicolumn{2}{|c|}{ Número de frutos } & \multicolumn{2}{|c|}{ Produção } \\
\hline & \multicolumn{2}{|c|}{ Método de propagação } & \multicolumn{2}{|c|}{ Método de propagação } & \multicolumn{2}{|c|}{ Método de propagação } \\
\hline & MP & EST & MP & EST & MP & EST \\
\hline Testemunha & $15,45 \mathrm{Ab}$ & $12,84 \mathrm{Bb}$ & $37,13 \mathrm{Aa}$ & $16,69 \mathrm{Ba}$ & $1,42 \mathrm{Aa}$ & $0,26 \mathrm{Ba}$ \\
\hline Leve & $15,21 \mathrm{Bb}$ & $17,29 \mathrm{Aa}$ & $24,00 \mathrm{Ab}$ & $19,94 \mathrm{Aa}$ & $0,72 \mathrm{Ab}$ & $0,36 \mathrm{Aa}$ \\
\hline Drástica & $18,00 \mathrm{Aa}$ & $17,48 \mathrm{Aa}$ & $25,61 \mathrm{Ab}$ & $21,36 \mathrm{Aa}$ & $0,69 \mathrm{Ab}$ & $0,50 \mathrm{Ba}$ \\
\hline
\end{tabular}

Médias seguidas pela mesma letra minúscula na coluna ou maiúscula na linha não diferem pelo teste de Tukey com nível de significância de 5\%.

Tabela 5 - Peso médio dos frutos $(\mathrm{g}), \mathrm{pH}$, acidez titulável (meq $\mathrm{L}^{-1}$ ) e antocianinas totais (mg cianidina 3-glicosídeo/100 g amostra) de plantas de mirtileiro 'Briteblue' em diferentes formas de propagação, micropropagadas e por estaquia e diferentes intensidades de poda (testemunha, leve e drástica). Pelotas, RS, 2016.

Table 5 - Average weight of fruit (g), $p H$, titratable acidity (meq $L^{-1}$ ) and anthocyanins (mg cyanidin 3-glucoside/100 g sample) of 'Briteblue' blueberry plants in different forms of propagation, micro propagation and cuttings and different pruning intensities (control, light and drastically). Pelotas, RS, 2016.

\begin{tabular}{lcccc}
\cline { 2 - 5 } & $\begin{array}{c}\text { Peso médio } \\
\text { dos frutos }\end{array}$ & $\mathrm{pH}$ & $\begin{array}{c}\text { Acidez } \\
\text { titulável }\end{array}$ & $\begin{array}{c}\text { Antocianinas } \\
\text { totais }\end{array}$ \\
\hline Micropropagação & $0,99^{\mathrm{ns}}$ & $2,10^{\mathrm{ns}}$ & $10,60^{\mathrm{ns}}$ & $527,20 \mathrm{a}^{*}$ \\
Estaquia & $0,98^{\mathrm{ns}}$ & $1,97^{\mathrm{ns}}$ & $14,93^{\mathrm{ns}}$ & $412,07 \mathrm{~b}$ \\
\hline Testemunha & $0,98^{\mathrm{ns}}$ & $1,95^{\mathrm{ns}}$ & $14,02 \mathrm{a}$ & $430,31^{\mathrm{ns}}$ \\
Leve & $0,92^{\mathrm{ns}}$ & $2,06^{\mathrm{ns}}$ & $12,38 \mathrm{~b}$ & $489,40^{\mathrm{ns}}$ \\
Drástica & $1,06^{\mathrm{ns}}$ & $2,10^{\mathrm{ns}}$ & $11,90 \mathrm{~b}$ & $489,20^{\mathrm{ns}}$ \\
\hline \multicolumn{1}{c}{$\mathrm{CV}(\%)$} & 18,43 & 9,20 & 3,29 & 13,85 \\
\hline
\end{tabular}

*Significativo pelo teste de tukey ao nível de $5 \%$ de probabilidade de erro. ${ }^{\text {ns }}$ não significativo. 
onde o peso dos frutos variou significativamente com a intensidade de poda nas plantas e as podadas levemente, tiveram frutos $40 \%$ menores do que as plantas podadas drasticamente.

Verifica-se que para essa cultivar, a técnica de micropropagação favoreceu a obtenção de frutos com maior concentração de antocianinas totais, quando comparada com a estaquia. As culturas de células de plantas podem sintetizar grandes quantidades de metabólitos secundários dentro de um período de cultivo de duas semanas. Esse fato pode ser explicado pelo fato da cultura de tecidos de plantas favorecer a síntese de grandes quantidades de metabólitos secundários dentro de um curto período de tempo (FUMAGALI et al. 2008).

Na mesma Tabela é demonstrada a influência dos tratamentos em estudo para os valores de antocianinas totais nos frutos de mirtileiro cv. 'Briteblue', onde a intensidade de poda não influenciou no acúmulo desses compostos. Essa constatação se difere do esperado, já que há tendência de maiores concentrações de antocianinas em plantas com maior intensidade de poda, devido a maior exposição solar e ainda, devido a maior condição de estresse ocasionada por essa prática. Resultado esse que contraria os encontrados por RADÜNZ et al. (2014), que verificaram que a intensidade de poda influenciou os teores de antocianinas.

A cor e os sólidos solúveis dos frutos 'Briteblue' foram influenciados pelos dois fatores estudados (Tabela 6), diferentemente do encontrado por
SOUZA et al. (2014), que verificaram que a prática de poda teve pouca influência sobre a qualidade dos frutos avaliados, onde o teor de sólidos solúveis não foi alterado nas condições do experimento. Outro estudo que também pode ser comparado, é o de JORQUERA-FONTENA et al. (2014), onde a concentração total de sólidos solúveis nos frutos aumentou aproximadamente $9 \%$ em plantas podadas drasticamente. Porém, MACHADO et al. (2004) verificaram em trabalhos realizados em diferentes safras, que o teor de sólidos solúveis totais apresenta grande variação, uma das explicações é a oscilação climática que ocorre entre os anos.

Verifica-se que a cor dos frutos foi mais intensa em plantas micropropagadas sem poda e com poda leve, assim como obtidas por estaquia sem poda e com poda drástica. Em ambos os métodos de propagação, as plantas sem poda produziram frutos com maior coloração, provavelmente pela menor emissão de novos ramos, e consequentemente, maior luminosidade no interior da copa. Resultados opostos foram obtidos em relação aos sólidos solúveis dos frutos, onde plantas propagadas in vitro e podadas drasticamente, assim como, as obtidas por estaquia, sem poda, geraram valores mais expressivos, o que demonstra a diferença no desenvolvimento das plantas, de acordo com a forma que as mesmas foram propagadas.

Houve interação entre os fatores para as variáveis fenólicos totais e atividade antioxidante dos frutos (Tabela 7). Plantas micropropagadas sem

Tabela 6 - Cor $(\Delta \mathrm{E})$ e sólidos solúveis $(\%)$ de frutos oriundos de plantas de mirtileiro 'Briteblue' em diferentes formas de propagação, micropropagadas e por estaquia e diferentes intensidades de poda (testemunha, leve e drástica). Pelotas, RS, 2016.

Table 6 - Color ( $\triangle E)$ and soluble solids (\%) of fruits coming from plants of 'Briteblue' blueberry in different forms of propagation, micro propagation and cuttings and different pruning intensities (control, light and drastically). Pelotas, RS, 2016.

\begin{tabular}{|c|c|c|c|c|}
\hline \multirow{3}{*}{$\begin{array}{c}\text { Intensidade de } \\
\text { poda }\end{array}$} & \multicolumn{2}{|c|}{$\Delta \mathrm{E}$} & \multicolumn{2}{|c|}{ Sólidos solúveis } \\
\hline & \multicolumn{2}{|c|}{ Método de propagação } & \multicolumn{2}{|c|}{ Método de propagação } \\
\hline & Micropropagação & Estaquia & Micropropagação & Estaquia \\
\hline Testemunha & $1,18 \mathrm{Aa}$ & $1,52 \mathrm{Aa}$ & $14,07 \mathrm{Bb}$ & $16,33 \mathrm{Aa}$ \\
\hline Leve & $1,13 \mathrm{Aa}$ & $0,49 \mathrm{Bb}$ & $14,20 \mathrm{Ab}$ & $14,53 \mathrm{Aab}$ \\
\hline Drástica & $0,65 \mathrm{Bb}$ & $1,11 \mathrm{Aa}$ & $16,60 \mathrm{Aa}$ & $12,57 \mathrm{Bb}$ \\
\hline $\mathrm{CV}(\%)$ & \multicolumn{2}{|c|}{19,40} & \multicolumn{2}{|c|}{6,99} \\
\hline
\end{tabular}

Médias seguidas pela mesma letra minúscula na coluna ou maiúscula na linha não diferem pelo teste de Tukey com nível de significância de 5\%. 
Tabela 7 - Fenólicos totais (mg ácido clorogênico equivalente/100 g tecido) e atividade antioxidante ( $\mu \mathrm{g}$ trolox equivalente/g tecido) de frutos de plantas de mirtileiro 'Briteblue' em diferentes formas de propagação, micropropagadas e por estaquia e diferentes intensidades de poda (testemunha, leve e drástica). Pelotas, RS, 2016.

Table 7 - Total phenolics (mg chlorogenic equivalent $/ 100 \mathrm{~g}$ tissue acid) and antioxidant activity ( $\mu \mathrm{g}$ trolox equivalent/g tissue) of 'Briteblue' blueberry fruit plant in different forms of propagation, micro propagation and cuttings and different pruning intensities (control, light and drastically). Pelotas, RS, 2016.

\begin{tabular}{|c|c|c|c|c|}
\hline \multirow{3}{*}{$\begin{array}{c}\text { Intensidade de } \\
\text { poda }\end{array}$} & \multicolumn{2}{|c|}{ Fenólicos totais } & \multicolumn{2}{|c|}{ Atividade antioxidante } \\
\hline & \multicolumn{2}{|c|}{ Método de propagação } & \multicolumn{2}{|c|}{ Método de propagação } \\
\hline & MP & EST & MP & EST \\
\hline Testemunha & $819,76 \mathrm{Aa}$ & $651,83 \mathrm{Ba}$ & $9635,30 \mathrm{Aa}$ & $7667,89 \mathrm{Bb}$ \\
\hline Leve & $644,09 \mathrm{Ab}$ & $695,41 \mathrm{Aa}$ & $7937,2 \mathrm{Bb}$ & $9033,6 \mathrm{Aa}$ \\
\hline Drástica & $671,09 \mathrm{Ab}$ & $677,09 \mathrm{Aa}$ & $7760,2 \mathrm{Bb}$ & $9504,43 \mathrm{Aa}$ \\
\hline $\mathrm{CV}(\%)$ & \multicolumn{2}{|c|}{8,92} & \multicolumn{2}{|c|}{7,03} \\
\hline
\end{tabular}

Médias seguidas pela mesma letra minúscula na coluna ou maiúscula na linha não diferem pelo teste de Tukey com nível de significância de 5\%.

poda e plantas obtidas por estaquia com podas leve e drástica, produziram frutos com maior teor de fenóis e antioxidantes. A maior presença desses compostos nas plantas propagadas in vitro e sem poda, pode ser justificada pois nas podadas drasticamente, ocorreu uma grande emissão de ramificações vegetativas e que consequentemente, conferem um maior sombreamento no interior da planta e, provavelmente por essa razão, não ocorreu diferenças no teor de antocianinas. O mesmo foi constatado por RIIHINEN et al. (2008), onde o menor dossel vegetativo permitiu maior incidência da luz solar sobre a superfície do fruto, visto que maiores níveis de radiação solar estão relacionados ao acréscimo de compostos fenólicos, principalmente antocianinas.

Diante deste contexto, conclui-se que plantas micropropagadas de mirtileiro 'Briteblue' apresentam maior crescimento vegetativo quando comparadas com plantas oriundas por estaquia e, além disso, a poda drástica favorece o desenvolvimento de ramos vegetativos de maior comprimento. Em relação as características produtivas, os frutos de plantas propagadas pela técnica de estaquia apresentam maior teor de antocianinas e aqueles colhidos em plantas sem poda ou com poda leve possuem maior atividade antioxidante.

\section{AGRADECIMENTOS}

A Coordenação de Aperfeiçoamento de Pessoal de Nível Superior (CAPES) pelo apoio financeiro e a Universidade Federal de Pelotas (UFPel) e Embrapa Clima Temperado pelas condições fornecidas para a execução do trabalho.

\section{REFERÊNCIAS}

BRACKMANN A et al. 2010. Armazenamento de mirtilo 'Bluegem' em atmosfera controlada e refrigerada com absorção e inibição de etileno. Revista Ceres 57:6-11.

DAMIANI CR \& SCHUCH MW. 2009. Diferentes substratos e ambientes no enraizamento in vitro de mirtilo. Ciência Rural 39:563-566.

FUMAGALI E et al. 2008. Produção de metabólitos secundários em cultura de células e tecidos de plantas: o exemplo dos gêneros Tabernaemontana e Aspidosperma. Revista Brasileira de Farmacognosia 18:627-641.

GIOVANELLI G \& BURATTI S. 2009. Comparison of polyphenolic composition and antioxidant activity of wild Italian blueberries and some cultivated varieties. Food Chesmistry 112:903-908.

JORQUERA-FONTENA E et al. 2014. Pruning severity affects yield, fruit load and fruit and leaf traits of 'Brigitta' blueberry. Journal of Soil Science and Plant Nutrition 14:855-868.

KADIR S. 2003. Why fruit trees fail to bear. Kansas: Kansas State University. $4 \mathrm{p}$.

LITWIÑCZUK W et al. 2005. Field performance of 
highbush blueberries (Vaccinium xcorymbosum L.) cv. 'Herbert' propagated by cuttings and tissue culture. Scientia Horticulturae 106:162-169.

MACHADO NP et al. 2004. Conservação pós-colheita de mirtilos Flórida, Woodard e Bluegem em atmosfera com oxigênio ionizado. II Simpósio Nacional do Morango e I Encontro de Pequenas Frutas e Frutas Nativas do Mercosul. In: Anais... p.300-304.

MARINO SR et al. 2014. Vegetative growth of three southern highbush blueberry cultivars obtained from micropropagation and softwood cuttings in two Florida locations. HortScience 49:556-561.

MORAES JO et al. 2007. Estudo do mirtilo (Vaccinium ashei Reade) no processamento de produtos alimentícios. Ciência e Tecnologia de Alimentos 27:18-22.

PESCIE $M$ et al. 2011. Effect of time and intensity of pruning on the yield and fruit quality of southern highbush blueberries (Vaccinium corymbosum) var. O'neal in Buenos Aires province. Revista de Investigaciones Agropecuarias 37:268-274.

RADÜNZ AL et al. 2014. Intensidade de poda na produção e na qualidade dos frutos de mirtileiro. Revista Brasileira de Fruticultura 36:186-191.

RIIHINEN $\mathrm{K}$ et al. 2008. Organ-specific distribution of phenolic compounds in bilberry (Vaccinium myrtillus) and 'northblue' blueberry (Vaccinium corymbosum $\mathrm{x} V$. angustifolium). Food Chemistry 110:156-160.

SCHUCH MW et al. 2007. AIB e substrato na produção de mudas de mirtilo cv. "Climax" através de microestaquia. Ciência Rural 37:1446-1449.

SOUZA ALK et al. 2011. Desempenho de mudas de mirtilo obtidas por micropropagação ou estaquia. Pesquisa Agropecuária Brasileira 46:868-874.

SOUZA ALK et al. 2014. Produção e qualidade de frutos de mirtileiros sob diferentes intensidades de poda. Ciência Rural 44:2157-2163.

SPIERS JM et al. 2002. Effects of pruning on 'Climax' Rabbiteye Blueberry. Acta Horticulturae 574:133-237.

TREVISAN R et al. 2008. Enraizamento de estacas herbáceas de mirtilo: influência da lesão na base e do ácido indolbutírico. Ciência e Agrotecnologia 32:402-406.

VIZZOTTO M. 2012. Propriedades funcionais das pequenas frutas. Informe Agropecuário 33:84-88. 